\title{
DNMT3A Low-Expression is Correlated to Poor Prognosis in Childhood B Cell Precursor Acute Lymphoblastic Leukemia and Confers Resistance to Daunorubicin on Leukemic Cells
}

\section{Weijing Li ( $\square$ lwj1985@126.com )}

Beijing Children's Hospital https://orcid.org/0000-0002-5860-0696

Shuguang Liu

Beijing Children's Hospital

Chanjuan Wang

Beijing Children's Hospital

Lei Cui

Beijing Children's Hospital

Xiaoxi Zhao

Beijing Children's Hospital

Wei Liu

Zhengzhou Children's Hospital

\section{Ruidong Zhang}

Beijing Children's Hospital

\section{Zhigang Li}

Beijing Children's Hospital

\section{Primary research}

Keywords: Childhood B-ALL, DNMT3A expression, Genome editing of DNMT3A, DNR drug resistance,

Posted Date: April 12th, 2021

DOl: https://doi.org/10.21203/rs.3.rs-193259/v1

License: (c) (i) This work is licensed under a Creative Commons Attribution 4.0 International License. Read Full License 


\section{Abstract}

\section{Background}

Little is known about DNMT3A expression and its prognostic significance in childhood B cell precursor acute lymphoblastic leukemia (BCP-ALL).

\section{Methods}

We determined DNMT3A mRNA expression in 102 children with BCP-ALL. Correlations with relapse-free survival (RFS) and common clinical characteristics were analyzed. DNMT3A was stably knocked out by CRISPR/Cas9 gene editing technology in 697 cell line. Cell proliferation activity after treated with daunorubicin was determined by CCK8 assay in DNMT3A KO 697 cell line.

\section{Results}

DNMT3A expression in BCP-ALL patients who were in CCR was higher than in those who got relapse $(P=0.0111)$. Receiver operating characteristic curve showed prognostic significance of DNMT3A expression $(P=0.003)$. Low expression of $D N M T 3 A(<0.197)$ was significantly correlated with poor RFS $(P<0.001)$ in children with BCP-ALL. Knock-out of DNMT3A in 697 cell line significantly increased IC50 of daunorubicin $(P=0.0057)$, indicating elevated resistance to daunorubicin.

\section{Conclusions}

Low expression of DNMT3A associates with poor prognosis in children with BCP-ALL. Knock-out of DNMT3A confers resistance to daunorubicin on leukemic cells.

\section{Introduction}

B cell precursor acute lymphoblastic leukemia (BCP-ALL) is the most common childhood malignancy. Although cure rate of childhood BCP-ALL has been greatly improved with risk-adjusted therapy [1, 2], relapsed leukemia is still a leading cause of death for children mainly due to therapy resistance [2-4]. Thus, it is of importance to clarifying the mechanisms of therapy resistance and relapse of BCP-ALL.

DNA methyltransferase 3A (DNMT3A) catalyzes de novo DNA methylation and plays important roles in the pathogenesis of malignancies including leukemia. Furthermore, DNMT3A mutations in acute myeloid leukemia (AML) and T cell ALL are associated with poor prognosis of the patients [5-11]. Our previous studies have shown that DNMT3A mutations can be found in a few of children with BCP-ALL, and are correlated with poor prognosis [12]. However, the expression level of DNMT3A and its prognostic significance in BCP-ALL remains unclear.

In this study, we assessed the relationship between expression level of DNMT3A and prognosis in Chinese childhood BCP-ALL. Moreover, CRISPR/Cas9 has been used to knock out DNMT3A gene in 
leukemic 697 cell line in order to explore the role of $D N M T 3 A$ expression playing in resistance to chemotherapeutic drugs. We showed that low expression of DNMT3A was correlated with poor treatment outcome, knock-out of this gene resulted in obvious resistance to daunorubicin (DNR), a common chemotherapeutic drug in treatment of ALL.

\section{Materials And Methods}

\section{Patients}

A total of 102 BCP-ALL patients with available diagnostic bone marrow (BM) samples were enrolled in this study. These patients were diagnosed and treated in accordance with the Chinese Children's Leukemia Group ALL 2008 Protocol (CCLG-ALL 2008) from July 2010 to May 2014 at Beijing Children's Hospital [4]. There were 64 boys and 38 girls, aged from 1 to 13 years with a median age of 4 . Thirty-four patients carried 4 types of fusion genes including TEL-AML1, E2A-PBX1, BCR-ABL, and TLS-ERG. The details of stratification and treatment according to CCLG-ALL 2008 were described previously $[4,13]$. Ninety-four patients were in continuous complete remission (CR), 8 patients relapsed 2 to 62 months after diagnosis. The follow-up time ranged from 1 to 92 months (median, 59 months). Additionally, BM samples from 11 patients at continuous $\mathrm{CR}$ were collected and used as control.

MRD at d33 (the end of induction of remission) and d78 (before consolidation therapy) were detected using RQ-PCR targeted at Ig/TCR (immunoglobulin and $T$ cell receptor gene rearrangements) according to European MRD laboratory guidelines [14-17].

Informed consents were obtained from all the children's parents or legal guardians.

\section{Cell lines}

Human BCP-ALL cell line 697, as a kind gift from Dr. Suning Chen at the first affiliated Hospital of Soochow University (Suzhou, China), was cultured in RPMI 1640 (GIBCO, USA) supplemented with 10\% fetal bovine serum (FBS, AusGeneX, Brisbane) and 1\% penicillin/streptomycin. HEK293T cell, kindly provided by Dr. Fen Chang at Peking University Health Science Center (Beijing, China), was cultured in DMEM supplemented with $10 \%$ FBS and $1 \%$ penicillin/streptomycin. All cells were maintained at $37^{\circ} \mathrm{C}$ in a humidified atmosphere containing 5\% $\mathrm{CO} 2$.

\section{Nucleic acid extraction}

Mononucleated cells were separated from $1 \mathrm{ml}$ of patients' BM aspirate by centrifugation with Ficoll 400 (MD Pacific Technology CO., Ltd.) and stored at $-70^{\circ} \mathrm{C}$ until use. Total RNA of samples was extracted and reverse transcribed using Trizol Reagent (Invitrogen, USA) and MMLV reverse transcriptase (Promega, USA) according to the manufacturers' instructions respectively. Genomic DNA of 697 cell line was extracted using a Blood \& Cell Culture DNA Midi Kit (TIANGEN, China) according to the manufacturer's protocol. 
Real-time quantitative polymerase chain reaction (RQ-PCR) was performed using Power SYBR ${ }^{\text {TM }}$ Green PCR Master Mix (Applied Biosystems 4367659) by an ABI Prism 7500 Sequence Detection System (Applied Biosystems, Foster City, CA, USA). GUS ( $\beta$-Glucuronidase) expression was used as an internal control. The cycling condition included pre-denaturation at $95^{\circ} \mathrm{C}$ for 30 seconds, followed by 40 cycles of $5 \mathrm{~s}$ at $95^{\circ} \mathrm{C}, 30 \mathrm{~s}$ at $55^{\circ} \mathrm{C}$ and $30 \mathrm{~s}$ at $72^{\circ} \mathrm{C}$. Primers are shown in Table 1 . We used the cDNA samples obtained from 697 cell line as a calibrator. The relative expression of DNMT3A was calculated by the method of $2^{-\triangle \triangle C t}$. The levels of DNMT3A and GUS were tested in triplicates.

Table 1

Oligo sequences

\begin{tabular}{|c|c|c|}
\hline Oligo name & Sequence & Description \\
\hline $\begin{array}{l}\text { DNMT3A ex7 } \\
\text { sg F }\end{array}$ & CACCGGGGGCCCGGGGAGTCTCAGA & \multirow[t]{2}{*}{ sgRNA primer } \\
\hline $\begin{array}{l}\text { DNMT3A ex7 } \\
\text { sg R }\end{array}$ & AAACTCTGAGACTCCCCGGGCCCCC & \\
\hline $\begin{array}{l}\text { DNMT3A ex7 } \\
\text { F }\end{array}$ & TTTCACGGCAAGGCAGCTGGTTG & \multirow[t]{2}{*}{ PCR primer (445bp) for T7e1 assay } \\
\hline $\begin{array}{l}\text { DNMT3A ex7 } \\
\text { R }\end{array}$ & AGAGGAGAGCAGGACGGGAGGAG & \\
\hline $\begin{array}{l}\text { DNMT3A } \\
\text { ex23 F }\end{array}$ & GCCACCTCTTCGCTCCGCTG & \multirow[t]{2}{*}{ RQ-PCR primer(239bp)for clinical samples } \\
\hline $\begin{array}{l}\text { DNMT3A } \\
\text { ex23 R }\end{array}$ & GATGATGTCCAACCCTTTTCGCAA & \\
\hline GUS F & GAAAATATGTGGTTGGAGAGCTCATT & \multirow{2}{*}{$\begin{array}{l}\text { RQ-PCR primer(101bp)as internal control } \\
\text { for clinical samples }\end{array}$} \\
\hline GUS R & CCGAGTGAAGATCCCCTTTTTA & \\
\hline
\end{tabular}

\section{Lenti DNMT3A-sgRNA-Cas9 constructs}

The cDNA sequence encoding sgRNA which targets a conserved sequence in exon 7 of human DNMT3A gene was synthesized and subcloned into LentiCRISPR-v2 plasmid (Addgene 52961, kindly provided by Dr. Jian Huang at Temple University, Philadelphia, PA) to make the lentiDNMT3A-sgRNA-Cas9 constructs. Briefly, the forward and reverse primers including 20 bp target DNMT3A sequence and Bsmbl sticky end were annealed and inserted into the lentiCRISPR-v2 plasmid digested with FastDigest Esp3I (Thermo Fisher Scientific, \#FD0454) (Fig. 1). sgRNA primer sequences have been reported by Gundry MC et al. previously and are shown in Table 1 [18].

\section{Lentivirus production and infection}


To produce lentivirus, $6.0 \mu \mathrm{g}$ of transfer plasmid lentiDNMT3A-sgRNA-Cas9 or control plasmid lentiCRISPR-v2 were co-transfected into HEK 293T cells with $4.5 \mu \mathrm{g}$ of packaging plasmids psPAX2 (AddGene 12260) and 3.0 $\mathrm{gg}$ of VSV-G (AddGene 8454) using FuGENE® 6 Transfection Reagent (Promega E2692) according to the manufacturer's instructions. After incubation for $48 \mathrm{~h}$ or $72 \mathrm{~h}$, the culture supernatants containing lentivirus were harvested and filtered with $0.45 \mu \mathrm{m}$ filter and stored at $-80^{\circ} \mathrm{C}$. The 697 cell line $\left(5 \times 10^{5}\right)$ was infected with the lentivirus at an M.O.I. of 40 , using spin-transduction (centrifuging the plate coated with $8 \mu \mathrm{g} / \mathrm{ml}$ polybrene (SANTA CRUZ) at $1200 \mathrm{~g}$ for 2 hours at $25^{\circ} \mathrm{C}$ ), then were cultured for 24 hours in the incubator. On the next day, the medium was changed with fresh RPMI 1640 complete medium and the cells were cultured for another 24 hours.

\section{T7EN1 assays for quantifying frequencies of indel mutations}

Lentivirus-infected cells were selected by $1 \mu \mathrm{g} / \mathrm{ml}$ puromycin for 2 days. Genomic DNA was extracted and used to amplify the genomic region flanking the DNMT3A sgRNA target site with KAPA2G Robust HotStart ReadyMix (KAPA BIOSYSTEMS KK5702) and PCR primers listed in Table 1. Then T7EN1 assay was performed using T7 Endonuclease I (NEB \#M0302L) according to the Instruction Manual. The digested DNA was analyzed on electrophoresis system using a $2 \%$ agarose gel.

\section{Western blotting}

A fraction of lentivirus-infected cells was lysed in NE-PER® Nuclear and Cytoplasmic Extraction Reagents (Thermo Fisher Scientific, USA). The lysates were denatured in $5 \times$ SDS loading buffer by boiling at $95^{\circ} \mathrm{C}$ for 10 minutes and were subjected on a NuPAGE ${ }^{\mathrm{TM}} 4-12 \%$ Bis-Tris Protein Gels (Invitrogen). After transferred to Biotrace NT nitrocellulose Transfer Membrane (PALL, 66485), the expression of proteins was detected using following antibodies: DNMT3A (D23G1) Rabbit mAb (CST 3598), DNMT3A (D2H4B) Rabbit mAb (CST 32578), Lamin B1 Mouse mAb (proteintech 66095-1-lg), Goat anti-Mouse lgG $(\mathrm{H}+\mathrm{L}$ ) Highly Cross-Adsorbed Secondary Antibody, Alexa Fluor 680 (Invitrogen A21058),Goat anti-Rabbit IgG (H + L) Highly Cross-Adsorbed Secondary Antibody, Alexa Fluor Plus 800 (Invitrogen A32735). The bands were scanned by LICOR Odyssey CLX.

\section{Cell viability}

The lentivirus-infected 697 cell line was plated into 96-well plate, $10^{4} /$ well. After treatment with $100 \mu \mathrm{l}$ $\operatorname{DNR}(0.005,0.01,0.05,0.1,0.5,1 \mu \mathrm{g} / \mathrm{ml})$, the cells were cultured for $48 \mathrm{~h}$ at $37^{\circ} \mathrm{C}$ in a humidified atmosphere containing $5 \% \mathrm{CO}_{2}$. Twenty microliters of Cell Counting Kit-8 (CCK-8, Yeasen 40203ES60*, Shanghai, China) solution were added to each well and mixed gently. After incubation for $1 \mathrm{~h}$, optical density (OD) at $450 \mathrm{~nm}$ was determined using a Spectra MAX 190 microplate reader. After calibrated with non-cellular background, cell viability was calculated using a non-treatment control regarded as $100 \%$ of cell viability.

\section{Statistical analysis}


Receiver Operating Characteristic (ROC) curve was used to decide the cut-off value of low- and highexpression of DNMT3A (DNMT3A $A^{\text {low }}$ and DNMT3A high) in leukemic cells of children with BCP-ALL. Fisher's exact test was used to test the differences in clinical characteristics and relapse rates between $D N M T 3 A^{\text {low }}$ and DNMT3 $A^{\text {high }}$ patients. Relapse free survival (RFS) was defined as the date of leukemia diagnosis to the date of recurrence. Survival estimates were calculated using the Kaplan-Meier method, and the groups were compared using the log-rank test. The independent prognostic significance of DNMT3A expression and the common clinical features was analyzed by Cox proportional hazards model (Method: Enter). All data were analyzed with the SPSS 16.0 software package and a $P$ value $<0.05$ was considered statistically significant. The fitting curves of inhibitory effects of DNR on cell proliferation were plotted by GraphPad Prism 8, and half maximal inhibitory concentration (IC50) was also calculated by the software.

\section{Results}

\section{DNMT3A expression in childhood BCP-ALL}

Firstly, we determined DNMT3A expression in 102 newly diagnosed (ND) BCP-ALL patients and 11 patients with non-malignant hematological disease (control) by relative quantitative PCR. As a result, DNMT3A expression in ND patients with BCP-ALL, ranged from 0.0006594 to 1.713 with a median of 0.4363 , was significantly higher than that in control patients (range: 0.08055 to 0.1865 , median: 0.1147 ; $P=0.0004$, Fig. 2a). Interestingly, DNMT3A expression in ND BCP-ALL patients who got relapse was significantly decreased compared with that in patients who were in continuous complete remission (CCR) at the last follow-up $(P=0.0111$, Fig. $2 b)$.

\section{Low expression of DNMT3A indicated poor prognosis in ND BCP-ALL patients}

ROC curve was used to examine the prognostic value of DNMT3A expression in BCP-ALL patients. It was found that the area under curve (AUC) was 0.819 ( $P=0.003$, Fig. 3a), which indicated that $D N M T 3 A$ expression could be a potential prognostic biomarker for ND BCP-ALL patients.

A cut-off of 0.197 was chosen as both the sensitivity (75.0\%) and the specificity (87.2\%) were acceptable. We then divided 102 patients into two groups, 22 cases with low DNMT3A expression $(\leq 0.197$, $D N M T 3 A^{\text {low }}$ ) and 80 cases with high $D N M T 3 A$ expression ( $\left.>0.197, D N M T 3 A^{\text {high }}\right)$. There was a significantly higher relapse rate in $D N M T 3 A^{\text {low }}$ group (6 out of 22 vs 2 out of 88 , Fisher's exact test, $P=$ 0.001). Moreover, poor RFS was observed in the patients of $D N M T 3 A^{\text {low }}$ group $(P<0.001)$ (Fig. $\left.3 b\right)$.

Furthermore, Cox proportional hazards regression analysis indicated the independent prognostic significance of $D N M T 3 A$ expression with common prognostic factors as multivariates (HR $=19.195,95 \%$ Cl: $3.159 \sim 116.651, P=0.001$, Table 2). These findings indicated that low expression of DNMT3A in leukemic cells at diagnosis could be a useful indicator for disease relapse in childhood BCP-ALL. 
Table 2

The independent prognostic significance of DNMT3A expression and other common clinical features

\begin{tabular}{|lllll|}
\hline Features & Hazard ratio (HR) & $P$ & \multicolumn{2}{l|}{$95 \%$ Cl for HR } \\
\cline { 4 - 5 } & & & Lower & Upper \\
\hline DNMT3A expression & 19.195 & 0.001 & 3.159 & 116.651 \\
\hline Age & 0.000 & 0.988 & 0.000 & \\
\hline WBC & 1.353 & 0.738 & 0.231 & 7.929 \\
\hline TEL-AML1 & 0.252 & 0.093 & 0.51 & 1.258 \\
\hline E2A-PBX1 & 1.025 & 1.000 & 0.000 & \\
\hline BCR-ABL1 & 1.446 & 1.000 & 0.000 & \\
\hline MRD at day33 & 3.517 & 0.212 & 0.487 & 25.380 \\
\hline MRD at day78 & 0.000 & 0.996 & 0.000 & \\
\hline
\end{tabular}

Comparison of clinical features between DNMT3 $A^{\text {low }}$ and DNMT3 $3 A^{\text {high }}$ patients

In the next step, we analyzed the correlation of DNMT3A expression with common clinical characteristics such as age, gender, white blood cell (WBC) count at diagnosis and fusion genes. However, no correlation was found between DNMT3A expression and above clinical characteristics (Table 3). 
Table 3

Correlation of DNMT3A expression with clinical characteristics in BCP-ALL

\begin{tabular}{|c|c|c|c|}
\hline & DNMT3A'low, $\mathrm{n}(\%)$ & DNMT3A ${ }^{\text {high }}, \mathrm{n}(\%)$ & $P$ \\
\hline \multicolumn{4}{|l|}{ Age } \\
\hline$<1$ or $\geq 10$ & $1(4.5)$ & $6(7.5)$ & \multirow[t]{2}{*}{1.000} \\
\hline $1 \sim 10$ & $21(95.5)$ & $74(92.5)$ & \\
\hline \multicolumn{4}{|l|}{ Gender } \\
\hline Male & $16(72.7)$ & $48(60)$ & \multirow[t]{2}{*}{0.327} \\
\hline Female & $6(27.3)$ & $32(40)$ & \\
\hline \multicolumn{4}{|c|}{ WBC $\left(* 10^{9} / L\right)$} \\
\hline$<50$ & $15(68.2)$ & $68(85)$ & \multirow[t]{2}{*}{0.118} \\
\hline$\geq 50$ & $7(31.8)$ & $12(15)$ & \\
\hline \multicolumn{4}{|c|}{ Fusion gene } \\
\hline Negative & $19(86.4)$ & $49(61.25)$ & \multirow[t]{5}{*}{0.264} \\
\hline$T E L-A M L 1$ & $3(13.6)$ & $25(31.25)$ & \\
\hline$E 2 A-P B X 1$ & $0(0)$ & $3(3.75)$ & \\
\hline$B C R-A B L 1$ & $0(0)$ & $2(2.5)$ & \\
\hline$T L S-E R G$ & $0(0)$ & $1(1.25)$ & \\
\hline \multicolumn{4}{|l|}{ MRD at d33 } \\
\hline$<10^{-3}$ & $18(85.7)$ & $67(89.3)$ & \multirow[t]{2}{*}{0.701} \\
\hline$\geq 10^{-3}$ & $3(14.3)$ & $8(10.7)$ & \\
\hline \multicolumn{4}{|l|}{ MRD at d78 } \\
\hline$<10^{-4}$ & $21(100)$ & $72(96)$ & \multirow[t]{2}{*}{1.000} \\
\hline$\geq 10^{-4}$ & $0(0)$ & $3(4)$ & \\
\hline
\end{tabular}

We further analyzed the association of DNMT3A expression with MRD at d33 and MRD at d78 respectively, but no significant correlation between them was found (Fisher's exact test, $P>0.05$, Table 3 ).

Knock-out of DNMT3A enhanced resistance of 697 cell line to DNR 
To confirm the correlation of low expression of DNMT3A with poor prognosis of children with BCP-ALL, firstly, we disrupted DNMT3A in 697 cell line. T7 endonuclease I (T7EN1) assay showed high efficiency of the sgRNA to direct Cas9-mediated ablation of DNMT3A (Fig. 4a). Furthermore, as expected, Western blotting indicated that $D N M T 3 A$ expression was remarkably reduced after infection with $D N M T 3 A-s g R N A$ lentivirus (Fig. 4b).

DNR is one of the main chemotherapeutic drugs in induction therapy of BCP-ALL. We next tested whether knock-out of DNMT3A gene could cause 697 cell line to be tolerant to DNR by CCK8 assay. These cells were treated by different concentrations of DNR for 24h. It was shown that IC50 was significantly increased in the DNMT3A-knockout cells, indicating decreased cell viability (Fig. 5, Control vs. DNMT3A $\mathrm{KO}, 0.06449$ vs. $0.1052 \mu \mathrm{g} / \mathrm{ml}, P=0.0057)$. These results demonstrated that sgRNA mediated Cas 9 knock-out of DNMT3A can causes 697 cell line to be resistant to DNR, implying that DNMT3A expression plays an important role in the sensitivity of leukemic cells to chemotherapeutic drugs such as DNR.

\section{Discussion}

In recent two decades, DNMT3A mutations have been found in approximately $20 \%$ of adult AML patients, $9 \%$ of adult T-ALL and $0 \sim 1.4 \%$ of childhood AML, and the hotspots of mutations are mainly located in exon 23 which encodes the catalytic methyltransferase domain [5-11]. DNMT3A mutations are associated with poor prognosis and used for risk stratification in AML [5-10], and is associated with increased age and adverse outcome in adult T-ALL [11]. However, few studies focused on the role of DNMT3A in BCP-ALL. Our previous study has shown that DNMT3A mutations can be found in exon 23 and its adjacent intron regions in a few of children with BCP-ALL $(5 / 182,2.7 \%)$, and may have adverse impact on prognosis [12].

As there are only a few BCP-ALL patients with DNMT3A mutations, we sought to determine the prognostic significance of DNMT3A expression in BCP-ALL. As expected, low expression leading to decreased methyltransferase activity was associated with relapse in 102 patients with BCP-ALL. Furthermore, knockout of DNMT3A increased IC50 of DNR in 697 cell line, indicating the relationship of low expression of $D N M T 3 A$ and chemoresistance.

It was reported that Dnmt3a loss in HSCs leads to hypomethylation of genes with a causal role in cancer, such as Runx1 and Gata3. Runx1 promotes murine erythroid progenitor proliferation and inhibits differentiation by preventing Pu.1 downregulation [19]. Gata3 targets Runx1 in the embryonic hematopoietic stem cell niche [20]. Thus, previous studies and ours suggest that deletion or lowexpression of Dnmt3a result in differentiation inhibition of HSCs and allow HSCs to be propagated indefinitely in vivo [21-24], which may play an important role in leukemogenesis and resistance to chemotherapy. This may provide us with an alternative target of therapy for childhood BCP-ALL.

It has been reported that DNMT3A expression is directly transactivated by transcription factor WT1 in Wilms' tumor cells [25] and is negatively regulated by p53 at the transcriptional level in lung cancer[26]. In fact, overexpression of the WT1 transcript was demonstrated in children with B-ALL at diagnosis [27], 
which may contribute to increased expression of DNMT3A in leukemic cells. However, increased expression of TP53 by 2 to 20 -fold higher in pediatric primary B-ALL than in healthy controls [28] would inhibit DNMT3A expression. Thus, the regulation mechanism of DNMT3A expression is quite complicated in different types of cancer cells and needs to be clarifed especially in childhood BCP-ALL.

In summary, we associated low expression of DNMT3A with poor prognosis in Chinese pediatric patients with B-ALL and resistance to DNR. Furthermore, successful disruption of DNMT3A in 697 cell line may facilitate the studies on mechanism of relapse and chemotherapeutic resistance for childhood BCP-ALL.

\section{Declarations}

Ethics approval and consent to participate: Yes

Consent for publication: Yes

Availability of data and materials: Emails to corresponding author Zhigang Li

\section{Competing interests}

The authors report no conflicts of interest. The authors alone are responsible for the content and writing of the paper.

\section{Funding}

This study was supported by grants from the Beijing Natural Science Foundation of China [No. 7194263 and 7202044]; the National Natural Science Foundation of China [No. 81870114 and 81970135], the Scientific Research Common Program of Beijing Municipal Commission of Education [No.

KM201810025025], the Beijing Municipal Science \& Technology Commission [No. Z181100001718100], the National Science and Technology Major Project of the Ministry of Science and Technology of China (No. 2017ZX09304029003).

\section{Authors' contributions}

Zhigang Li designed this research and revised the manuscript $₫$ Weijing Li performed experiments, interpreted/analyzed data, prepared figures and wrote the manuscript. Chanjuan Wang also performed part of the experiments and Lei Cui helped in analyzing data and preparing figures. Wei Liu and Ruidong Zhang also contributed to study design and organized informed consent. Shuguang Liu and Xiaoxi Zhao contributed patient material and provided clinical data.

\section{Acknowledgments}

The authors would like to thank all staff members in the hematology oncology center and in the laboratory of hematologic diseases who have taken care of the patients and given the help for the studies. 


\section{References}

1. Maloney KW, et al. Outcome in Children With Standard-Risk B-Cell Acute Lymphoblastic Leukemia: Results of Children's Oncology Group Trial AALL0331. J Clin Oncol. 2020;38(6):602-12.

2. Hunger SP, Mullighan CG. Acute Lymphoblastic Leukemia in Children. N Engl J Med. 2015;373(16):1541-52.

3. Kimura S, Mullighan CG. Molecular markers in ALL: Clinical implications. Best Pract Res Clin Haematol. 2020;33(3):101193.

4. Cui $L$, et al. Outcome of children with newly diagnosed acute lymphoblastic leukemia treated with CCLG-ALL 2008: The first nation-wide prospective multicenter study in China. Am J Hematol. 2018;93(7):913-20.

5. Park DJ, et al. Characteristics of DNMT3A mutations in acute myeloid leukemia. Blood Res. 2020;55(1):17-26.

6. Li W, et al. DNMT3A mutations in Chinese childhood acute myeloid leukemia. Med (Baltim). 2017;96(31):e7620.

7. Brunetti L, Gundry MC, Goodell MA. DNMT3A in Leukemia. Cold Spring Harb Perspect Med, 2017. $7(2)$.

8. Ho PA, et al. Leukemic mutations in the methylation-associated genes DNMT3A and IDH2 are rare events in pediatric AML: a report from the Children's Oncology Group. Pediatr Blood Cancer. 2011;57(2):204-9.

9. Yang L, Rau R, Goodell MA. DNMT3A in haematological malignancies. Nat Rev Cancer. 2015;15(3):152-65.

10. Shah MY, Licht JD. DNMT3A mutations in acute myeloid leukemia. Nat Genet. 2011;43(4):289-90.

11. Bond J, et al. DNMT3A mutation is associated with increased age and adverse outcome in adult Tcell acute lymphoblastic leukemia. Haematologica. 2019;104(8):1617-25.

12. Li W, et al. DNMT3A mutations and prognostic significance in childhood acute lymphoblastic leukemia. Leuk Lymphoma. 2015;56(4):1066-71.

13. Gao C, et al. Clinical features, early treatment responses, and outcomes of pediatric acute lymphoblastic leukemia in China with or without specific fusion transcripts: a single institutional study of 1,004 patients. Am J Hematol. 2012;87(11):1022-7.

14. Szczepanski T, et al. Vdelta2-Jalpha rearrangements are frequent in precursor-B-acute lymphoblastic leukemia but rare in normal lymphoid cells. Blood. 2004;103(10):3798-804.

15. van Dongen JJ, et al. Design and standardization of PCR primers and protocols for detection of clonal immunoglobulin and T-cell receptor gene recombinations in suspect lymphoproliferations: report of the BIOMED-2 Concerted Action BMH4-CT98-3936. Leukemia. 2003;17(12):2257-317.

16. van der Velden $\mathrm{VH}$, van Dongen JJ. MRD detection in acute lymphoblastic leukemia patients using Ig/TCR gene rearrangements as targets for real-time quantitative PCR. Methods Mol Biol. 2009;538:115-50. 
17. Cui $L$, et al. Combined analysis of minimal residual disease at two time points and its value for risk stratification in childhood B-lineage acute lymphoblastic leukemia. Leuk Res. 2010;34(10):1314-9.

18. Gundry MC, et al. Highly Efficient Genome Editing of Murine and Human Hematopoietic Progenitor Cells by CRISPR/Cas9. Cell Rep. 2016;17(5):1453-61.

19. Willcockson MA, et al. Runx1 promotes murine erythroid progenitor proliferation and inhibits differentiation by preventing Pu.1 downregulation. Proc Natl Acad Sci U S A. 2019;116(36):17841-7.

20. Fitch SR, et al. Gata3 targets Runx1 in the embryonic haematopoietic stem cell niche. IUBMB Life. 2020;72(1):45-52.

21. Jeong M, et al. Loss of Dnmt3a Immortalizes Hematopoietic Stem Cells In Vivo. Cell Rep. 2018;23(1):1-10.

22. Gu T, et al. DNMT3A and TET1 cooperate to regulate promoter epigenetic landscapes in mouse embryonic stem cells. Genome Biol. 2018;19(1):88.

23. Zhang $X$, et al. DNMT3A and TET2 compete and cooperate to repress lineage-specific transcription factors in hematopoietic stem cells. Nat Genet. 2016;48(9):1014-23.

24. Challen GA, et al. Dnmt3a is essential for hematopoietic stem cell differentiation. Nat Genet. 2011;44(1):23-31.

25. Szemes $M$, et al. Control of epigenetic states by WT1 via regulation of de novo DNA methyltransferase 3A. Hum Mol Genet. 2013;22(1):74-83.

26. Lin RK, Wang YC. Dysregulated transcriptional and post-translational control of DNA methyltransferases in cancer. Cell Biosci. 2014;4:46.

27. Chen JS, et al. Comparison of minimal residual disease (MRD) estimated by flow cytometry and by real-time quantitative PCR of Wilms tumor gene 1 (WT1) transcript expression in children with acute lymphoblastic leukemia. Leuk Res. 2007;31(10):1351-7.

28. Oh L, et al. Expression of p53 N-terminal isoforms in B-cell precursor acute lymphoblastic leukemia and its correlation with clinicopathological profiles. BMC Cancer. 2020;20(1):110.

\section{Figures}




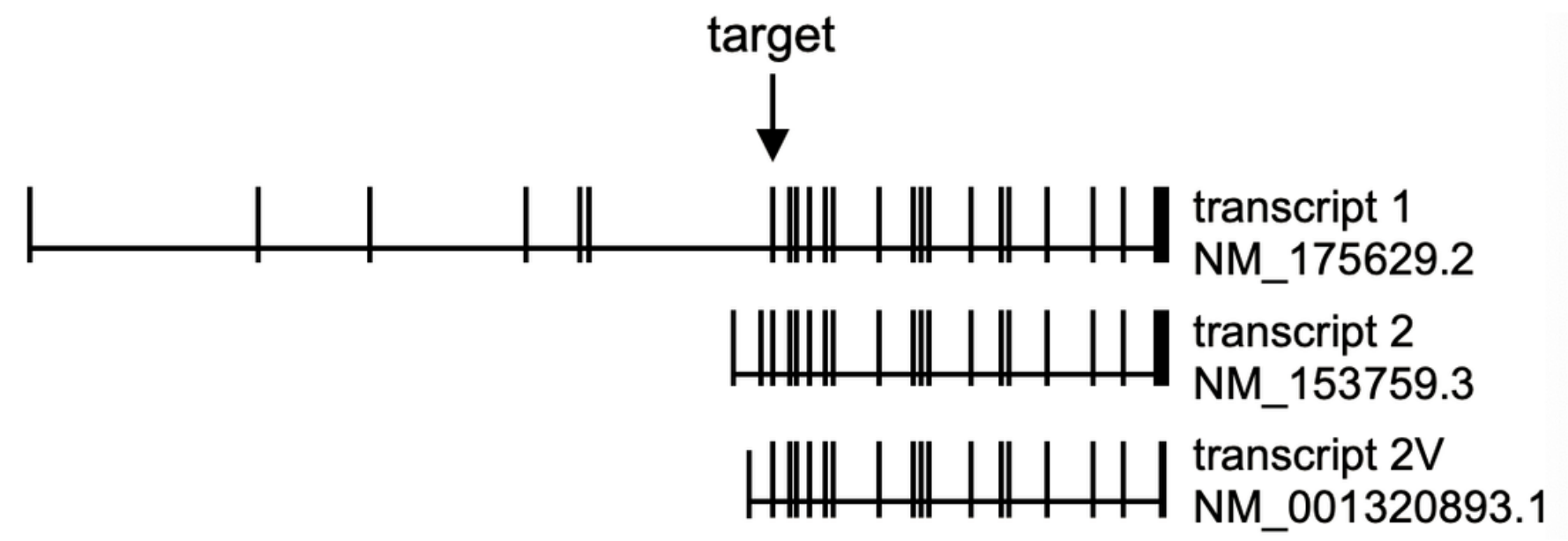

A

lentiCRISPR v2

(Plasmid \#52961)

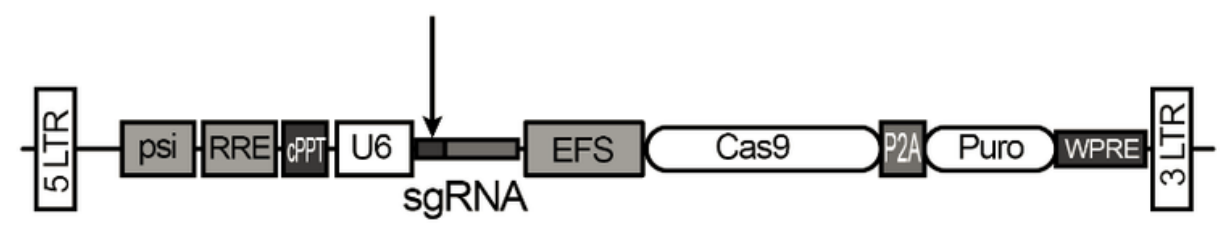

B

Figure 1

Schematic diagram of sgRNA targeting DNMT3A. a. The structure of DNMT3A gene and the three common transcripts. Black vertical lines: exons. Horizontal lines: introns. Arrow: the location of sgRNA targeting exon 7. b. The structure of lentiCRISPR v2 plasmid. The arrows indicate the sgRNA sequence. 

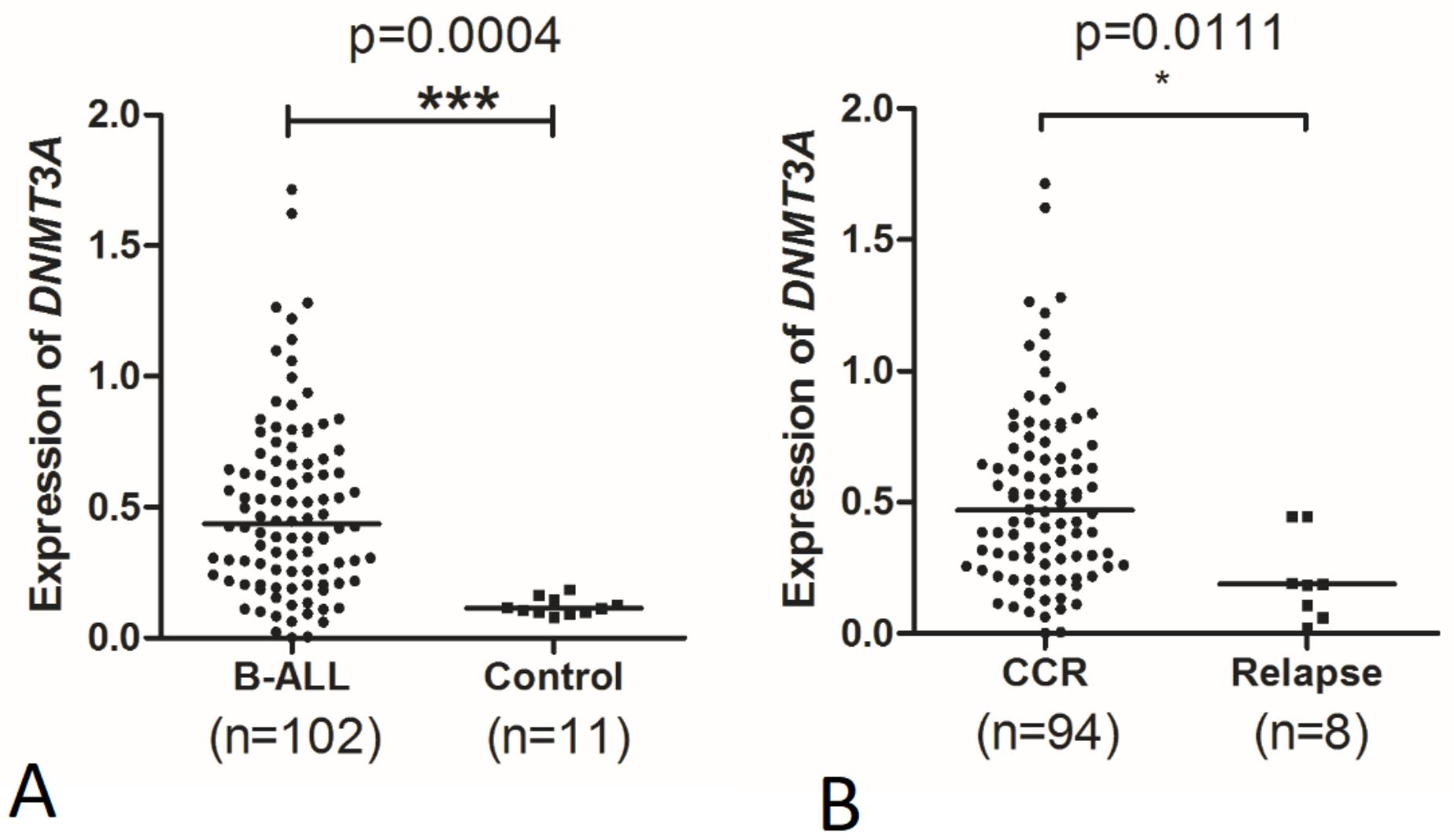

Figure 2

DNMT3A expression in ND BCP-ALL patients and controls. a. DNMT3A expression was significantly increased in ND BCP-ALL patients compared with that of controls. b. DNMT3A expression was significantly decreased in ND BCP-ALL patients who relapsed compared with that in ND patients in CCR. 


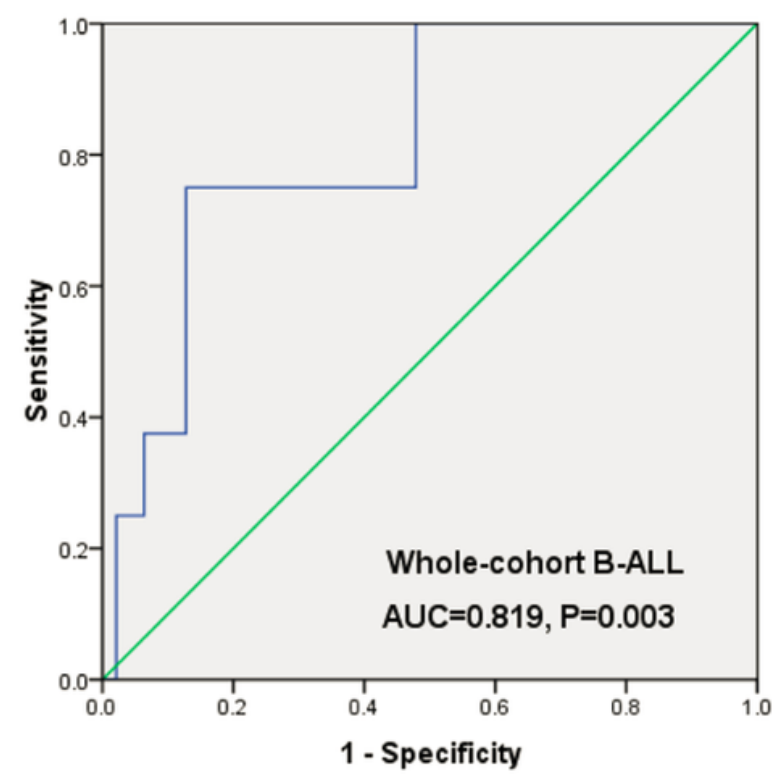

A

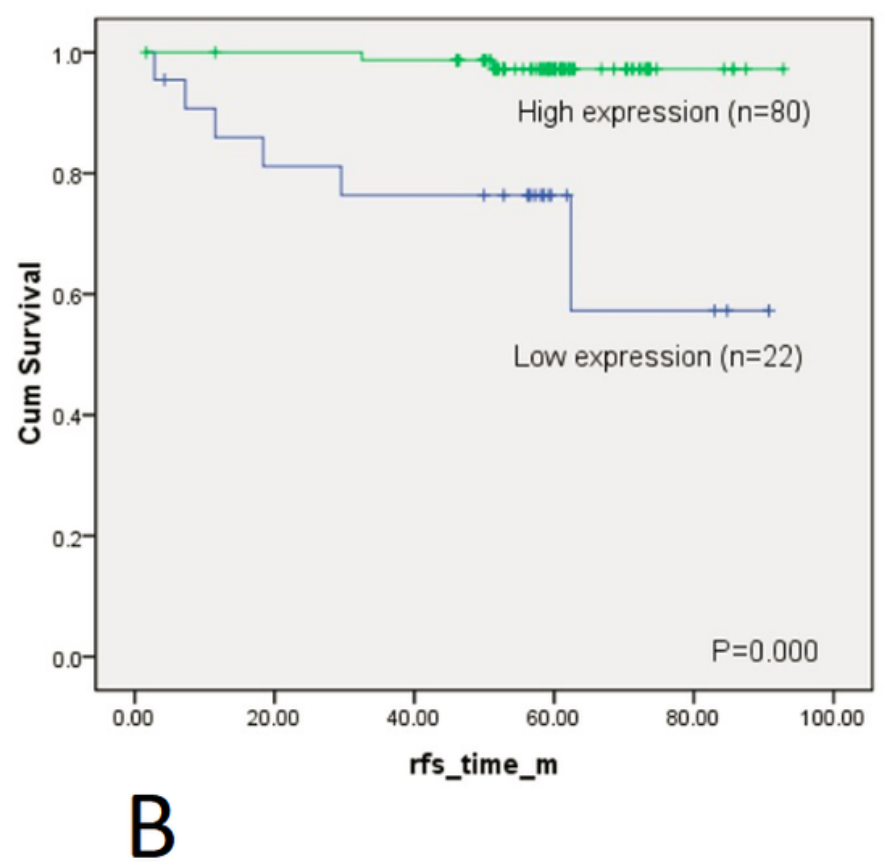

Figure 3

Prognostic significance of DNMT3A expression in 102 children with BCP-ALL. a. ROC curve analysis of DNMT3A expression with relapse as an event. B. The patients in DNMT3Alow group had poorer RFS than those in DNMT3Ahigh group $(P<0.001)$.

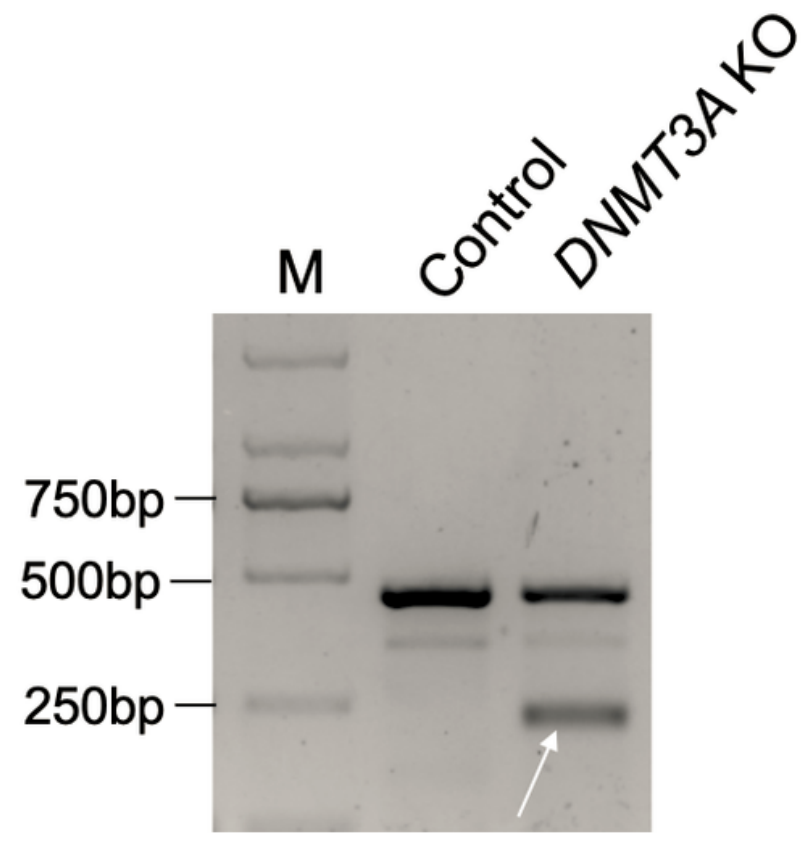

A

DNMT3A1
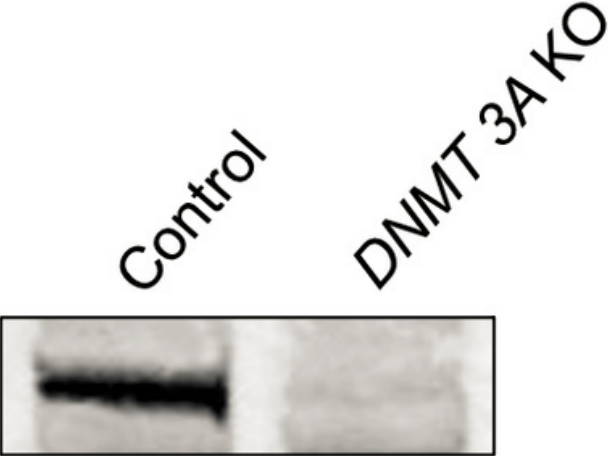

DNMT3A2

DNMT3A2V

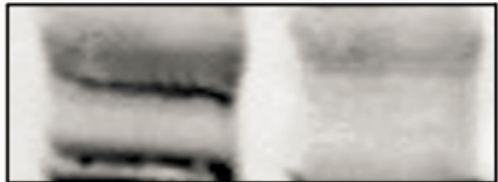

LAMINB

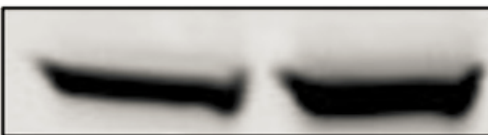

B

Figure 4 
LentiCRISPR/Cas9 mediated editing of DNMT3A gene in 697 cell line. a. T7e1 assay analysis of specific sgRNA-mediated indels at DNMT3A locus in 697 cell line. The lower migrating bands marking by a white arrow represent the disrupted gene alleles. b. Expression of three DNMT3A protein variants was significantly reduced in 697 cell line infected by DNMT3A-sgRNA lentivirus.

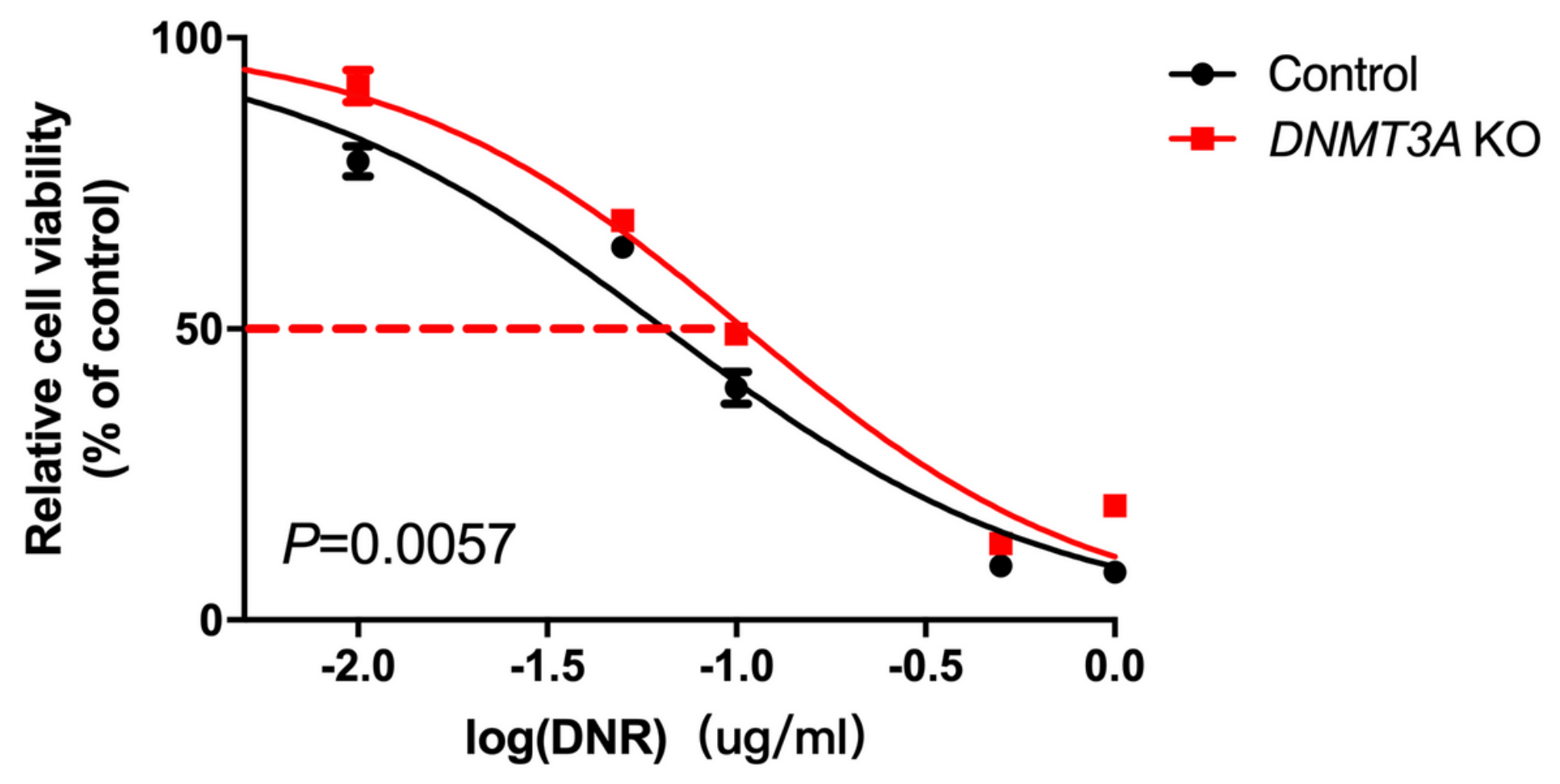

Figure 5

Knock-out of DNMT3A gene increased resistance of 697 cell line to DNR. IC50 of DNR significantly increased in DNMT3A-knockout cells (Independent-samples T test, $P=0.0057)$. The standard errors of the means are shown ( $n=3$ experiments for each drug concentration). 